\title{
TITLE:
}

\section{On Reduction-Based Process Semantics}

AUTHOR(S):

Honda, Kohei; Yoshida, Nobuko

\section{CITATION:}

Honda, Kohei ...[et al]. On Reduction-Based Process Semantics. 数理解 析研究所講究録 1995, 902: 10-25

ISSUE DATE:

1995-03

URL:

http://hdl.handle.net/2433/59391

RIGHT: 


\title{
On Reduction-Based Process Semantics
}

\author{
Kohei Honda Nobuko Yoshida \\ Department of Computer Science, Keio University \\ 3-14-1 Hiyoshi, Kohoku-ku, Yokohama, 223, Japan
}

\begin{abstract}
A formulation of semantic theories for processes which is based on reduction relation and equational reasoning is studied. The new construction can induce meaningful theories for processes, both in strong and weak settings. The resulting theories in many cases coincide with, and sometimes generalise, observation-based formulation of behavioural equivalence. The basic construction of reduction-based theories is studied, taking a simple name passing calculus called $\nu$-calculus as an example. Results on other calculi are also briefly discussed.
\end{abstract}

\section{Introduction}

The aim of the present paper is to investigate a general formulation of process semantics which can induce canonical congruence over agents solely based on reduction relation and equational reasoning. Our starting point is the formulation of operational semantics for processes based on reduction relation and structural rules, introduced by Berry and Boudol [3] and Milner [14]. By regarding structural rules as the embedded algebra for processes (cf. $\alpha$-equality), we can view reduction relation as representing the basic mechanism of computation in a given formalism. Especially in the area of higher-order process calculi $[13,14,15,8]$, it was found that the reduction-based formulation enjoys much more simplicity than the transition-based one. It remains open, however, whether we can have a general framework to derive meaningful process equivalences from the new construction, which are as significant as well-known behavioural equivalences over agents e.g. bisimilarities [19, 12].

What we are going to show in the subsequent sections, is that a general framework to derive significant congruences over processes from the new construction in a mathematically elegant way, does exist. While works with the aim similar to ours appeared already $[16 ; 4]$, our construction has several significant aspects not found in those precursors. Firstly, the basic formal apparatus is an extension of the well-studied method in both strict and lazy $\lambda$-theories $[2,1,18]$, using the maximality condition among a certain family of congruences to derive canonical equality over agents. Specifically a fundamental element of the construction is reduction-closure for equality, which generalises $\beta$-equality into the stateful regime of concurrent processes. The congruence relations over processes obtained by the method turn out to be behaviourally significant in varied process calculi, attaining intuitively sound notions of equality over agents. Secondly, one methodological appeal of the construction in the present paper is that we obtain those

\footnotetext{
* Appeared in Proc. of 13th FST/TCS, LNCS 761, pp.371-387, Springer-Verlag, 1993. Revised
} in July 1994. 
canonical theories without committing ourselves to a specific notion of observation we even do not employ convergence predicate in $[16,4]$, which is considered to be the "minimised observability" in [16]. Such development is important when it is difficult to identify a canonical notion of observation in given formalisms and programming languages (e.g. [11, 20, 13, 8]). A significant example is $\nu$-calculus $[8,7,10]$, an offspring from $\pi$-calculus [13], where we have two "natural" notions of observation which induce quite different transition relations and bisimilarities. Semantics for this small calculus is taken as the main object of study in the present exposition, showing that a behaviourally significant equality over agents is obtained in our reduction-based framework. Results on other calculi are also mentioned at the end.

The structure of the paper follows. Section 2 gives a brief introduction to $\nu$-calculus as far as necessary in the present paper. Section 3 develops basic construction of reductionbased equality for $\nu$-calculus. Section 4 further studies the reduction-based equational construction for $\nu$-calculus, restoring usual weak and strong bisimilarities in a purely reduction-based framework. Section 5 mentions results on other calculi, gives comparison with related work, and points out further issues.

Most of proofs are omitted, for which the reader would refer to [9].

\section{2 -calculus}

2.1. Terms and reduction. $\nu$-calculus, an offspring from $\pi$-calculus $[6,13,14]$, is a small formalism of concurrency with the notion of asynchronous name passing as the interaction primitive $[8,7,10]$ ( $\mathrm{cf}$. [4]). The simple primitive, coupled with capability to generate new names, gives $\nu$-calculus enough power to construct versatile structures of interaction $[8,10]$. Below we introduce basic notions of the calculus as far as needed in the present exposition.

Let $\mathbf{N}$ be a countable set of names and $\mathbf{V}$ be a countable set of (name) variables, ranged over by $a, b, c, .$. and by $x, y, z, .$. , respectively. $u, v, w, .$. range over their union, the set of identifiers. The set of $\nu$-terms $\mathbf{T}_{\nu}$, ranged over by $P, Q, \ldots$, is given by the following grammar.

$$
P::=\leftarrow u v \mid \text { ux.P }|P, Q| a \triangleright P|\operatorname{lux.P}| \Lambda
$$

Among terms, " $\leftarrow u v$ " denotes a message to a target $u$ carrying a value $v$, while " $u x . P$ " denotes a receptor which receives a message at $u$ and instantiates the value in $P$. In $u x . P$, $x$ binds free occurrences of $x$ in $P$ (just like $\lambda x . M$ ). " $P, Q$ " is a concurrent composition of $P$ and $Q$. " $a>P$ " is a scope restriction where the initial $a$ binds its free occurrences in $P . ! u x . P$ is a lazy replicator which replicates its body each time it interacts, where $x$ is a binding occurrence. " $\Lambda$ " is a syntactic convention to denote nothing.

Free (resp. bound) names in $P$ is denoted by $\mathcal{F N}(P)$ (resp. $\mathcal{B N}(P)$ ). Free (resp. bound) variables ${ }^{2}$ in $P$ is denoted by $\mathcal{F} \mathcal{V}(P)$ (resp. $\mathcal{B V}(P)$ ). We also assume the usual notion of (multiple) substitution, written $\{\tilde{v} / \tilde{u}\}$ where $\tilde{u}$ and $\tilde{v}$ are strings of identifiers (names or variables) with the same length and all identifiers in $\tilde{u}$ are distinct. $\sigma, \sigma^{\prime}$ etc. range over substitutions. $\equiv_{\alpha}$ denotes $\alpha$-conversion. Closed and open terms are defined as

\footnotetext{
${ }^{2}$ For discussions on variables for names, see [9].
} 
usual (with respect to name variables). Thus $\leftarrow a b$ and $a x . \leftarrow x b$ are closed but $\leftarrow y c$ and $a x$. $\leftarrow c z$ are open. Then, for an open term $P, \sigma$ from variables to names, is a closing substitution iff $\mathcal{F} \mathcal{V}(P \sigma)=\emptyset$.

Some conventions: $a b \triangleright P$ denotes $a \triangleright(b \triangleright P)$; "," is the weakest in association, e.g. $a \triangleright P, Q \stackrel{\text { def }}{=}(a \triangleright P), Q$, and associates to the left; we write $\leftarrow c$ and c.P to mean we do not care the value to be communicated.

Reduction relation provides the basic notion of computing in the formalism. To formulate reduction we first stipulate a set of structural rules following Milner [14] (cf. $[3]$ ). We define $\equiv$ to be the smallest congruence relation over $\nu$-terms generated by the following rules.

(i) $P \equiv Q$ if $P \equiv_{\alpha} Q$

(ii) $P, Q \equiv Q, P \quad(P, Q), R \equiv P,(Q, R) \quad P, \Lambda \equiv P$

(iii) $a a \triangleright P \equiv a \triangleright P \quad a b \triangleright P \equiv b a \triangleright P \quad a \triangleright \Lambda \equiv \Lambda \quad a \gg P, Q \equiv a \triangleright(P, Q)$ if $a \notin \mathcal{F N}(Q)$.

Let $\partial, \partial^{\prime}, \ldots$ range over a sequence of concurrent composition of terms of the forms $\leftarrow u v, u x . P$ and !ux.P. The following simple definition of reduction relation relies on the fact that any $P$ can be transformed to a form $\tilde{c} \triangleright(\partial)$ using $\equiv$.

\section{DEFinition 2.1 (Reduction relation)}

(i) One-step reduction, denoted by $\rightarrow$, is the smallest relation over terms generated by the following rules.

$$
\begin{aligned}
& \text { COM: } \quad \tilde{c} \triangleright\left(\partial, \leftarrow u v, u x . P, \partial^{\prime}\right) \rightarrow \tilde{c} \triangleright\left(\partial, P\{v / x\}, \partial^{\prime}\right) \\
& \text { REP: } \tilde{c} \triangleright\left(\partial, \leftarrow u v, ! u x . P, \partial^{\prime}\right) \rightarrow \tilde{c} \triangleright\left(\partial, P\{v / x\}, ! u x . P, \partial^{\prime}\right) \\
& \text { STR: } \\
& \frac{P^{\prime} \equiv P, P \rightarrow Q, Q \equiv Q^{\prime}}{P^{\prime} \rightarrow Q^{\prime}}
\end{aligned}
$$

(ii) Multi step reduction $\rightarrow$ is defined: $\rightarrow \stackrel{\text { def }}{=} \rightarrow^{*} \cup \equiv$.

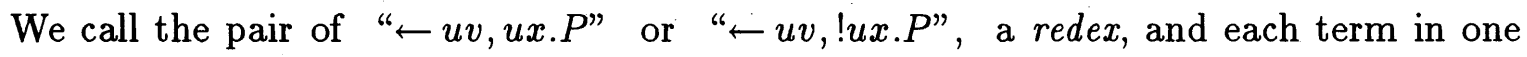
redex is called another's redex pair. Below we give some examples of reduction, along with several important expressions.

(i) Let $P \oplus Q \stackrel{\text { def }}{=} c \triangleright(\leftarrow c, c . P, c . Q)$ with $c$ fresh. Then: $P \oplus Q \rightarrow(P, c \triangleright c . Q)$ and $P \oplus Q \rightarrow(Q, c \gg c . P)$. Note $c \gg c . Q$ never reduces nor interacts.

(ii) Let $\mathcal{F W}(a b) \stackrel{\text { def }}{=} a y . \leftarrow b y . \quad$ Then: $\mathcal{F W}(a b), \leftarrow a c \rightarrow \leftarrow b c . \quad \mathcal{F} \mathcal{W}(a b)$ is called a forwarder.

(iii) Let $\mathcal{E Q}(a b) \stackrel{\text { def }}{=}(! \mathcal{F W}(a b), ! \mathcal{F W}(b a))$. Then: $\mathcal{E Q}(a b), \leftarrow a c \rightarrow \mathcal{E Q}(a b), \leftarrow b c \rightarrow$ $\mathcal{E} \mathcal{Q}(a b), \leftarrow a c \rightarrow \ldots \mathcal{E} \mathcal{Q}(a b)$ is called an equator. Note $\mathcal{E} \mathcal{Q}(a b) \equiv \mathcal{E} \mathcal{Q}(b a)$.

(iv) Let $\mathcal{I}(a) \stackrel{\text { def }}{=} ! \mathcal{F W}(a a)$. Then: $\mathcal{I}(a), \leftarrow a c \rightarrow \mathcal{I}(a), \leftarrow a c \rightarrow \ldots \mathcal{I}(a)$ is called an identity receptor.

(v) Let $\Omega \stackrel{\text { def }}{=} o \triangleright(\leftarrow o o, \mathcal{I}(o))$. Then: $\Omega \rightarrow \Omega \rightarrow \Omega \rightarrow \ldots$ 
2. 2. Labelled transitions and bisimilarities. Let $l, l^{\prime}, .$. range over labels given by:

$$
l=\tau|\downarrow a b| \uparrow a b \mid \uparrow a(b)
$$

where "(b)" is the only bound occurrence. We write $\mathcal{B N}(l)$ and $\mathcal{F N}(l)$ for the sets of bound and free names in $l$. One important notion concerning labels is relevance: a label $l$ is relevant to a pair of terms $P$ and $Q$ if $\mathcal{B N}(l) \cap(\mathcal{F N}(P) \cup \mathcal{F N}(Q))=\emptyset$. Now we give rules which induce transition relations over closed $\nu$-terms each of which represents a particular notions of observation. In the following, we assume $a, b \notin\{\tilde{c}\}$ in IN and $\mathrm{OUT}_{f}, a \notin\{\tilde{c}\} \wedge b \in\{\tilde{c}\}$ in $\mathrm{OUT}_{b}$, and each term as a whole is closed.

$$
\begin{aligned}
& \star \mathrm{IN}_{s}: \quad \tilde{c} \triangleright\left(\partial, a x . P, \partial^{\prime}\right) \stackrel{\downarrow a b}{\longrightarrow} \tilde{c} \triangleright\left(\partial, P\{b / x\}, \partial^{\prime}\right) \quad \mathrm{OUT}_{b}: \tilde{c} \triangleright\left(\partial, \leftarrow a b, \partial^{\prime}\right) \stackrel{\uparrow a(b)}{\longrightarrow} \tilde{c} \backslash \dot{v} \triangleright\left(\partial, \partial^{\prime}\right) \\
& \star \star \mathrm{IN}_{a}: \quad \tilde{c} \triangleright\left(\partial, \partial^{\prime}\right) \stackrel{\lfloor a b}{\longrightarrow} \tilde{c} \triangleright\left(\partial, \leftarrow a b, \partial^{\prime}\right) \quad \mathrm{OUT}_{f}: \quad \tilde{c} \triangleright\left(\partial, \leftarrow a b, \partial^{\prime}\right) \stackrel{\uparrow a b}{\longrightarrow} \tilde{c} \triangleright\left(\partial, \partial^{\prime}\right) \\
& \mathrm{COM}: \tilde{c} \triangleright\left(\partial, \leftarrow a b, a x . P, \partial^{\prime}\right) \stackrel{\tau}{\longrightarrow} \tilde{c} \triangleright\left(\partial, P\{b / x\}, \partial^{\prime}\right) \quad \text { STR: } \quad \frac{P^{\prime} \equiv P, P \stackrel{l}{\longrightarrow} Q, Q \equiv Q^{\prime}}{P^{\prime} \stackrel{l}{\longrightarrow} Q^{\prime}}
\end{aligned}
$$

Out of six rules four without marks are common to both transition relations, while one with $\star$ is for synchronous transition, and one with $\star \star$ is for asynchronous transition. Intuitively $\mathrm{IN}_{s}$ rule assumes the synchronization is essential in observation, while $\mathrm{IN}_{a}$ assumes just asynchronous exchanges of messages suffices. Two different transition relations are denoted by $\stackrel{l}{\longrightarrow}_{s}$ (the synchronous case) and $\stackrel{l}{\longrightarrow}$ (the asynchronous case). Note in both cases $\tau$ transition coincides with one-step reduction. We also define transition which ignores $\tau$ actions. $\stackrel{\hat{l}}{\Longrightarrow}_{s}\left(\right.$ resp. $\stackrel{\hat{l}}{\Longrightarrow}_{a}$ ) stands for $\rightarrow$ if $l=\tau$, else for $\longrightarrow \stackrel{l}{\longrightarrow} \rightarrow$ (resp. $\rightarrow \stackrel{l}{\longrightarrow} \rightarrow \longrightarrow$ ). Note if $P$ is closed then $P \stackrel{l}{\longrightarrow} P^{\prime}$ implies $P^{\prime}$ is again closed.

Then synchronous strong (resp. weak) bisimulation is any symmetric relation over closed $\nu$-terms, say $\mathcal{R}$, such that, if $P \mathcal{R} Q$, whenever $P \stackrel{l}{\longrightarrow} P^{\prime}$ with $l$ relevant w.r.t. $P$ and $Q$, there exists $Q^{\prime}$ such that $Q \stackrel{l}{\longrightarrow} Q^{\prime}$ (resp. $P \stackrel{\hat{l}^{\prime}}{\Longrightarrow} Q$ ) and $P^{\prime} \mathcal{R} Q^{\prime}$. The maximum relation among such relations exists and is called synchronous strong (resp. weak) bisimilarity, $\sim_{s} \quad\left(\right.$ resp. $\left.\approx_{s}\right)$. By changing $\stackrel{l}{\longrightarrow}_{s}$ into $\stackrel{l}{\longrightarrow} a$ and $\stackrel{\hat{l}}{\longrightarrow}_{s}$ into $\stackrel{\hat{l}}{\Longrightarrow}_{a}$, one gets asynchronous strong (resp. weak) bisimulation and asynchronous strong (resp. weak) bisimilarity, written $\sim_{a}\left(\right.$ resp. $\left.\approx_{a}\right)$. For open terms, we let $P \sim_{s} Q$ with $P$ or $Q$ open when $P \sigma \sim_{s} Q \sigma$ for any closing substitution $\sigma$, and similarly for other bisimilarities. Some properties of bisimilarities follow.

\section{Proposition 2.2}

(i) $\sim_{s}, \approx_{s}, \sim_{a}$ and $\approx_{a}$ are congruence relations ${ }^{3}$.

(ii) $\sim_{s}$ is a proper subset of $\sim_{a}$, and $\approx_{s}$ is a proper subset of $\approx_{a}$.

We note that a differentiating pair for the strong case is e.g. $\langle(\mathcal{I}(a), \Omega), \Omega\rangle$, and for weak case, $\langle\mathcal{I}(a), \Lambda\rangle$. Remark that reduction of $\mathcal{I}(a)$ in 2.1 shows $\mathcal{I}(a)$ does not give any effect to outside, though in fact it engages in interaction.

${ }^{3}$ As a consequence of introduction of name variables, we require a congruence over $\mathbf{T}_{\nu}$, say $\cong$, should satisfy: $P \cong Q \Rightarrow P\{v / x\} \cong Q\{v / x\}$ for any $v$ and $x$. 
Finally an important remark is that we cannot decide which of these two paradigms of observation is more "basic" to the calculus as far as we rely on labelled transition relation or related notions. While this suggests an essential merit of the notion of observables, i.e. applicability to varied purposes, for canonical treatment of computational formalisms, some universal construction is called for. Note also that the notion of convergence as employed in $[4,16]$ cannot be the answer since we get different equivalences according to whether we take "asynchronous convergence" (where we only take output into account) or "synchronous convergence" (where we take both input and output into account). This is not what we can decide within the convergence-based semantic framework. These considerations motivate the construction in the subsequent sections.

2.3. $\nu$-theories. Equation among $\nu$-terms is the central topic in the next two sections. While to directly deal with congruence relations among terms is possible, to treat equations as generated by a formal theory, is often convenient.

DEFINITION 2.3 A $\nu$-theory, or simply a theory, is a formal theory, their formulae of the form $P=Q$, with at least the following axioms and rules.

$\begin{array}{llll}\text { 1. } P=Q & \text { when } P \equiv Q & \text { 4. } P=Q \Rightarrow P, R=Q, R & \text { 7. } P=Q \Rightarrow u x . P=u x . Q\end{array}$

2. $P=Q \Rightarrow Q=P \quad$ 5. $P=Q \Rightarrow R, P=R, Q$ 8. $P=Q \Rightarrow$ !ux. $P=$ ! ux. $Q$

3. $P=Q, Q=R \Rightarrow P=R$ 6. $P=Q \Rightarrow c \gg P=c \gg Q$ 9. $P=Q \Rightarrow P\{v / x\}=Q\{v / x\}$

Rule 9 conveys the meaning of name variables. Some notations:

(i) $\Im, \Im^{\prime}$.. range over $\nu$-theories. The $\nu$-theory with no additional axioms and rules is denoted $\Im \equiv$.

(ii) If $P=Q$ is provable in $\Im$ then we write $\Im \vdash P=Q$, or simply $P=Q$ when the concerned theory is obvious from the context.

(iii) Given a set of equations $\mathcal{E}, \mathcal{E}+\Im$ is the result of adding equations as axioms to $\Im$. $\mathcal{E}+$ denotes $\mathcal{E}+\Im_{\equiv} . \Im+\Im^{\prime}$ is a result of adding the set of equations from two theories as axioms to the above rules ${ }^{4}$. We extend this to an arbitrary family of $\nu$-theories, writing $\sum\left\{\Im_{i}\right\}_{i \in I}, I$ being an index set.

(iv) The relation induced by a theory $\Im$ is denoted by $|\Im|$. Given a family of $\nu$ theories, the maximum (resp. minimum) theories are those whose corresponding relations are the maximum (resp. minimum) in that family. We say a $\nu$-theory $\Im$ is a subtheory of another theory $\Im^{\prime}$ if $|\Im| \subset\left|\Im^{\prime}\right|$. If the inclusion is strict then the former is a proper subtheory of the latter.

(v) We say a theory is consistent if it does not equate all possible pairs of terms. A theory is inconsistent if it is not consistent.

Given the above definition, we can form theories corresponding to bisimilarities presented already, by adding equations from each bisimilarity to the minimum theory. We denote them by $\Im_{\sim_{s}}, \Im_{\sim_{a}}, \Im_{\approx_{s}}$, and $\Im_{\approx_{a}}$. Note we have $\left|\Im_{\sim_{s}}\right|=\sim_{s}$ (by $\sim_{s} \supset \equiv$ and congruence of $\sim_{s}$ ) and similarly for other theories.

\footnotetext{
${ }^{4}$ Essentially the sum is the congruent closure of the union of congruences (or the lub in the lattice of congruences).
} 


\section{Basic Constructions}

3. 1. Reduction closure property. The notion of states is essential in concurrency. A term may change its meaning during its reduction so that "equality" in this setting means that two equated terms can go to an equated state again. That is, if two terms are ever equated and one reduces to any term, then another should be able to reduce to an equated term again. What we want here is that the notion of equation is in harmony with the notion of reduction relation. The following formalises the idea ${ }^{5}$.

DEFINITION 3.1 A $\nu$-theory $\Im$ is reduction-closed, if, whenever $\Im \vdash P=Q, P \rightarrow P^{\prime}$ implies, for some $Q^{\prime}, Q \rightarrow Q^{\prime}$ and $\Im \vdash P^{\prime}=Q^{\prime}$.

Note if we have $\beta$-equality, i.e. $P \rightarrow P^{\prime} \Rightarrow P=P^{\prime}$, the closure property is satisfied vacuously: thus the property generalises $\beta$-equality into the stateful regime (the usage of $\rightarrow$ instead of $\rightarrow$ is essential in this aspect; think of $I I={ }_{\beta} I$ with $I \stackrel{\text { def }}{=} \lambda x . x$ ). Also note that, by Definition 3.1, $\Im$ is reduction-closed if and only if, whenever $\Im \vdash P=Q$, $C[P] \rightarrow P^{\prime}$ implies, for some $Q^{\prime}, C[Q] \rightarrow Q^{\prime}$ with $\Im \vdash P^{\prime}=Q^{\prime}$, where $C[]$ is an arbitrary context. This makes one orientation of the above definition explicit, i.e. an aspect as a test on the internal consistency of an equational theory under arbitrary contexts, considering state changes during reduction. We shall often call reduction-closed $\nu$-theories simply reduction theories.

Before stating one important property of reduction theories, we need a small but useful lemma.

LEMMA 3.2 (Chain Lemma) Let $\Im^{\prime}$ be $\sum\left\{\Im_{i}\right\}_{i \in I}$, a sum of a family of $\nu$-theories and $\Im^{\prime} \vdash P=Q$. Then we have a chain of equations: $\Im_{j_{0}} \vdash P=R_{0}, . ., \Im_{j_{k}} \vdash R_{k-1}=$ $R_{k}, \ldots, \Im_{j_{n}} \vdash R_{n-1}=Q$ for some $j_{i} \in I, 0 \leq i \leq n$.

Using the lemma we get:

PROPOSITION 3.3 Let $\Im_{i}$ be reduction-closed for all $i \in I$. Then $\sum\left\{\Im_{i}\right\}_{i \in I}$ is also reduction-closed.

Note the proposition does not imply existence of the maximum consistent reductionclosed equality, since $\sum\left\{\Im_{i}\right\}_{i \in I}$ above can be inconsistent. In fact one unpleasant fact is that there is no maximum element in the family of consistent reduction-closed $\nu$-families (see 3.4). Moreover, though the reduction closure is intended to be the basic criteria for internal consistency of $\nu$-theories, equations in some reduction theories are pathological even if they are consistent. One of examples is $\Im_{\mathcal{G}} \stackrel{\text { def }}{=}\left\{(P, \mathcal{G})=(Q, \mathcal{G}) \mid P, Q \in \mathbf{T}_{\nu}\right\}+$ where $\mathcal{G} \stackrel{\text { def }}{=} g \triangleright \leftarrow g g$. Note that, since $\mathcal{G}$ is an utterly inactive term, an agent $(P, \mathcal{G})$ behaves just like $P$ in any context. Hence the fact that $\Im_{\mathcal{G}}$ equates $(P, \mathcal{G})$ and $(Q, \mathcal{G})$ for any $P$ and $Q$ implies that the theory absurdly identify any two behaviour solely because

${ }^{5}$ The closure property has a subtle yet crucial difference from a seemingly similar notion in $[16,15]$ by Milner and Sangiorgi. See 5.2 for discussions. 
both contain $\mathcal{G}$. At the same time, the theory can be easily shown to be reduction-closed, and consistency is immediate. There are infinitely many such theories.

Thus the reduction-closure property falls short of imposing satisfactory constraint on $\nu$-theories, both mathematically and intuitively. What further condition should we impose on $\nu$-theories, which should be as semantically neutral as possible and be faithful to reduction-based construction? Here we take the idea inspired by the equational scheme of sensible $\lambda$-theories, where identification of meaningless (unsolvable) terms internally filters off "unsound" equations, just because they would lead to inconsistency [2]. A similar equational scheme is found to be effective in the lazy $\lambda$-calculus, see $[1,18]$. Meaninglessness in our context, however, cannot be given by such notions as unsolvability or strong unsolvability which are specific to $\lambda$-calculi, but should be given in the way applicable to concurrency formalisms in general. The notion we introduce for the purpose is called insensitivity.

3. 2. Insensitivity and sound theories. Insensitive terms are those which never interact with their enclosing contexts. The notion is intended to give a sufficient condition for operational insignificance of a term in the weak regime. Below let $\mathcal{A N}(P)$ be defined as: $\mathcal{A N}(\leftarrow a b)=\mathcal{A N}(a x . P)=\mathcal{A N}(! a x . P)=\{a\}, \mathcal{A N}(a \triangleright P)=\mathcal{A N}(P) \backslash\{a\}$, $\mathcal{A N}(P, Q)=\mathcal{A N}(P) \cup \mathcal{A N}(Q)$, and $\mathcal{A N}(\Lambda)=\emptyset$.

Definition 3.4 A closed term $P$ is insensitive if, for all $P^{\prime}$ such that $P \rightarrow P^{\prime}$, we have $\mathcal{A N}\left(P^{\prime}\right)=\emptyset$. An open term is insensitive iff it is insensitive after any closing substitution. The set of insensitive terms is denoted by $\operatorname{lns}_{\nu}$.

Paraphrasing, an agent is insensitive if its subterm never participates in reduction with the outside (i.e. never becomes a part of a redex whose pair lies outside of the agent). It is easy to prove that $\operatorname{lns}_{\nu}$ is closed under structural rules, multi-step reduction, and name substitution. Some examples of (non-)insensitive terms follow.

EXAMPLE $3.5 \quad \Lambda$ and $\Omega$ are insensitive, but neither $\leftarrow a b, c \triangleright(\leftarrow c e, c x . \leftarrow b e)$ nor $\mathcal{I}(a)$ are insensitive.

The next proposition formally tells us that insensitive terms actually "do nothing." Let the set of reduction contexts ${ }^{6}$ be given by: $C_{r}[]::=[]\left|a \triangleright C_{r}[]\right|\left(C_{r}[], P\right) \mid\left(P, C_{r}[]\right)$. We let $C_{r}[], C_{r}^{\prime}[], .$. range over the set of contexts. Then we define a generic reduction of a context as: $C_{r} \rightarrow C_{r}^{\prime} \Leftrightarrow{ }_{\text {def }} \quad \forall P . C_{r}[P] \rightarrow C_{r}^{\prime}[P]$. When $C_{r} \rightarrow C_{r}^{\prime}$ (which reads $C_{r}$ generically reduces to $C_{r}^{\prime}$ ), the former context can reduce to the latter without any participation of the term in a hole. Using the idea we have:

\section{Proposition 3.6 (Characterization of insensitivity)}

$$
\forall P \in \operatorname{lns}_{\nu} . C_{r}[P] \rightarrow R \Rightarrow R \equiv C_{r}^{\prime}\left[P^{\prime}\right] \wedge C_{r} \rightarrow C_{r}^{\prime} \wedge P \rightarrow P^{\prime} \wedge P^{\prime} \in \operatorname{lns}_{\nu} .
$$

The proposition says that if an insensitive term is put in a reduction context, whatever reduction takes place in the configuration, we can collect no evidence that the term

\footnotetext{
${ }^{6}$ We can use ordinary contexts but the reduction-contexts make the formulation simpler without losing generality.
} 
in a hole has participated in the change of the configuration at all. Remark that the characterization is given solely in terms of reduction relation and contextual closure, thus telling us the behavioural insignificance of insensitive terms independently of any "observational commitment".

The fundamental mechanism we are going to introduce into our equational construction is the identification of insensitive terms in reduction-closed $\nu$-theories. Proposition 3.6 provides a good reason for the identification. Let us call consistent reduction theories which equate any two insensitive terms, 'sound. Among sound $\nu$-theories one finds:

PROPOSITION $3.7 \quad \Im_{\approx_{s}}$ and $\Im_{\approx_{a}}$ are both sound.

In contrast, $\Im_{\sim_{s}}$ and $\Im_{\sim_{a}}$ are not sound, though they are obviously reduction-closed (cf. 4.3 later).

3. 3. Intrinsic observables. An essential fact about sound $\nu$-theories is that sound $\nu$-theories are, a posteriori, automatically equipped with observables. The induced observables are behaviourally significant, and, as studied in depth in the next section, they well reflect important aspects of operations of the calculus. The obtained observables in turn bring about a certain canonical $\nu$-theory as we shall see soon.

Let a pair of terms be incompatible, written $P \# Q$, if for any sound $\Im, \Im \nvdash P=Q$. Some such pairs become useful in deriving observability.

Lemma $3.8 \leftarrow c \# \Lambda, c . \Lambda \# \Lambda$, and $(\leftarrow c, c . \Lambda) \# \Lambda$.

Proof. To prove $\leftarrow c \# \Lambda$, first we show $\Im \vdash \leftarrow c=\Lambda \Rightarrow \Im \vdash \leftarrow c^{\prime}=\Lambda$ for any $c^{\prime}$ with $\Im$ sound. Take a context: $C[] \stackrel{\text { def }}{=} c \triangleright\left(c . \leftarrow c^{\prime},[], \Lambda\right)$ with $c^{\prime} \neq c$. Then $C[\Lambda]=\Lambda$ while $C[\leftarrow c] \rightarrow c \triangleright\left(\leftarrow c^{\prime}, \Lambda\right)=\left(\leftarrow c^{\prime}, c \triangleright \Lambda\right)=\leftarrow c^{\prime}$. Since $\leftarrow c^{\prime} \nrightarrow$ we have $\leftarrow c^{\prime}=\Lambda$ for arbitrary $c^{\prime}$, as required. Next we show $\leftarrow c \# \Lambda$ for arbitrary c. Suppose $\Im \vdash \leftarrow c=\Lambda$ in some sound theory. Let us be given any $P \in \mathbf{T}_{\nu}$. Then we can always find some $c^{\prime}$ such that $c^{\prime} \notin \mathcal{F N}(P)$ (since $\mathcal{F N}(P)$ is always finite), and with this $c^{\prime}$ we construct a context $C[] \stackrel{\text { def }}{=} c^{\prime} \triangleright\left([], c^{\prime} . P\right)$. By assumption and the result above, we have $\leftarrow c^{\prime}=\Lambda$. Hence we should have $C[\Lambda]=C\left[\leftarrow c^{\prime}\right]$. But $C\left[\leftarrow c^{\prime}\right] \rightarrow c^{\prime} \triangleright P=c^{\prime} \triangleright(P, \Lambda)=\left(P, c^{\prime} \triangleright \Lambda\right)=P$ while $C[\Lambda]=\Lambda$ hence $P=\Lambda$ for an arbitrary $P$, contradiction. Hence $\leftarrow c \# \Lambda$. To prove $c . \Lambda \# \Lambda$, take $C[] \stackrel{\text { def }}{=}([], \leftarrow c)$ and reason similarly. For $(\leftarrow c, c . \Lambda) \# \Lambda$, take $C[] \stackrel{\text { def }}{=} c \triangleright(c . \leftarrow e,[])$ with $e$ fresh.

Other notable incompatible pairs are $(\leftarrow a, \leftarrow b) \oplus(\leftarrow a, \leftarrow c) \#(\leftarrow a,(\leftarrow b \oplus \leftarrow c))$, and $(\leftarrow a \# \leftarrow b)$ if $a \neq b$. The former example tells us how sound theories are near to the usual (weak) bisimulation.

We now formulate a notion of "generic observable" intrinsic in sound theories, by a simple transition system.

$$
\tilde{c} \triangleright\left(\partial, \leftarrow a b, \partial^{\prime}\right) \stackrel{\uparrow a}{\rightarrow} \tilde{c} \triangleright\left(\partial, \partial^{\prime}\right) \quad(a \notin\{\tilde{c}\}) \quad \frac{P \equiv P^{\prime} P \stackrel{\uparrow a}{\rightarrow} Q Q \equiv Q^{\prime}}{P^{\prime} \stackrel{\uparrow a}{\rightarrow} Q^{\prime}}
$$


Note the transition is only concerned with the output messages, and, as such, only their targets, not values. Based on the transition relation, we have one of the most essential results in the present paper.

$\underline{\text { THEOREM } 3.9}$ (Observability) Let $\Im$ be a sound $\nu$-theory and $\Im \vdash P=Q$. Then

$$
P \stackrel{\uparrow a}{\sim} P^{\prime} \Rightarrow Q \rightarrow \stackrel{\uparrow a}{\rightarrow} \rightarrow Q^{\prime} \quad \text { for some } Q^{\prime} \text { with } \Im \vdash P^{\prime}=Q^{\prime}
$$

Proof. First suppose $\Im \vdash P=Q$ for some sound theory $\Im$. We show if $P \stackrel{\uparrow a}{\sim} P^{\prime}$ then there should be some $Q^{\prime}$ such that $Q \rightarrow \stackrel{\uparrow a}{\rightarrow} \rightarrow Q^{\prime}$ with $\Im \vdash P^{\prime}=Q^{\prime}$, as far as $\Im$ is sound. Now take a context $C[] \stackrel{\text { def }}{=}(a . \leftarrow c, c . \Lambda,[])$ with $c$ fresh. Then easily $C[P] \rightarrow\left(\leftarrow c, c . \Lambda, P^{\prime}\right) \rightarrow P^{\prime}$. Since $\Im$ is reduction-closed, we should have, for some $Q^{\prime \prime}, C[Q] \rightarrow Q^{\prime \prime}$ such that $\Im \vdash P^{\prime}=Q^{\prime \prime}$. Now we show $Q \rightarrow \stackrel{\uparrow a}{\rightarrow \rightarrow Q^{\prime \prime}}$. This is established by showing that $Q^{\prime \prime}$ cannot contain a term $c . \Lambda$.

But suppose $c . \Lambda$ is in $Q^{\prime \prime}$. Then let $\tilde{b}$ be such that $\{\tilde{b}\} \supset \mathcal{F N}(P) \cup \mathcal{F N}(Q)$ and $c \notin\{\tilde{b}\}$. Since $P^{\prime}=Q^{\prime \prime}$. we have $\tilde{b} \triangleright P^{\prime}=\tilde{b} \triangleright Q^{\prime \prime}$. But since $Q^{\prime \prime}$ contains $c . \Lambda$ it either contains $\leftarrow c$ or $a . \leftarrow c$ concurrently composed with $c . \Lambda$ (note $c . \Lambda$ and $\leftarrow c$ as originally contained in $C[Q]$ can only interact with each other so $c$ cannot occur anywhere other than these terms during reduction). Hence $\tilde{b} \triangleright Q^{\prime \prime}=c . \Lambda$ if $a . \leftarrow c$ never interacts or, with some $Q^{\prime \prime \prime}, \tilde{b} \triangleright Q^{\prime \prime} \rightarrow \tilde{b} \triangleright Q^{\prime \prime \prime}$ where $\tilde{b} \triangleright Q^{\prime \prime \prime}=(c . \Lambda, \leftarrow c)$. But from Lemma 3.8 this is contradiction, hence $c . \Lambda$ can not be in $Q^{\prime \prime}$. But if $c . \Lambda$ is not in $Q^{\prime \prime}$ then there should be interaction between $a . \leftarrow c$ and a term in $Q$ so that we have $Q \rightarrow \stackrel{\uparrow a}{\rightarrow} \rightarrow Q^{\prime \prime}$ as required.

In the above proof, note how equation of two terms with discrepant behaviour results in inconsistency via the reduction-closure property and identification of insensitive terms. Thus soundness "generates sense" in equations. We also note that the result, which neglects input observable and values to carry, is basically the maximum observability we can derive from the soundness condition, see 4.1 and 4.2 .

3.4. The maximal theory. The essential technical consequence of observability is the existence of isolation sets. Let $\mathbf{T}$ be a proper subset of $\mathbf{T}_{\nu}$. Then we say a theory $\Im$ isolates $\mathbf{T}$, or $\mathbf{T}$ is an isolation set in $\Im$, if $\Im \vdash P=Q$ and $P \in \mathbf{T}$ then $Q \in \mathbf{T}$. Using Chain Lemma we easily obtain:

LEMMA 3.10 (Isolation Lemma) Let $\Im^{\prime}$ be $\sum\left\{\Im_{i}\right\}_{i \in I}$, a sum of a family of $\nu$-theories. Then if $\Im_{i}$ isolates $\boldsymbol{T}$ for all $i \in I$, then $\Im^{\prime}$ also isolates $\boldsymbol{T}$.

But Theorem 3.9 tells us a sound theory isolates a set $\{P \mid P \rightarrow \stackrel{\uparrow a}{\leftrightarrow \rightarrow}\}$ for each $a$, and isolation of some non-empty set implies that the theory is consistent. Hence we get:

Proposition 3.11 Let $\Im_{i}$ be sound for each $i \in I$. Then $\sum\left\{\Im_{i}\right\}_{i \in I}$ is also sound.

The existence of the maximum sound theory follows. 
Proposition 3.12 Let $P \cong Q \Leftrightarrow$ def $\Im \vdash P=Q$ for some sound $\Im$. We define: $\Im_{\nu}^{*} \stackrel{\text { def }}{=}\{(P=Q) \mid P \cong Q\}+$. Then $\Im_{\nu}^{*}$ is the maximum sound theory.

Note that, by the construction of sound theories, $\Im_{\nu}^{*}$ is maximal among the whole family of consistent reduction-closed theories. By $\left|\Im_{\mathcal{G}}\right| \not \subset\left|\Im_{\nu}^{*}\right|$, we also know there is no maximum element among the family. We also note that insensitive terms are not isolated in $\Im_{\nu}^{*}$, e.g. $\Im_{\nu}^{*} \vdash \mathcal{I}(a)=\Lambda$ (for the equation use $\left|\Im_{\nu}^{*}\right| \supset \approx_{a}$ ), unlike unsolvable terms in the $\lambda$-theories. This is natural since all we wanted to do is to start from the smallest semantic commitment to get the broadest meaningful equality over processes.

The following proposition suggests an interesting relationship between our framework and the theory in $[1,18]$. We use the convergence predicate in the asynchronous regime: $P \Downarrow \quad \Leftrightarrow_{d e f} \exists a . P \rightarrow \stackrel{\uparrow a}{i}$.

Proposition 3.13 (Behavioural characterization of $\left.\Im_{\nu}^{*}\right)$ We define relations $\simeq_{\kappa}(\kappa \in$ Ord) (the class of ordinals), by the following ordinal recursion:

$-P \simeq_{0} Q$ if $\forall C . C[P] \Downarrow \quad \Leftrightarrow \quad C[Q] \Downarrow$.

$-P \simeq_{\kappa+1} Q$ if $\forall C$.

$$
\begin{aligned}
& C[P] \rightarrow P^{\prime} \Rightarrow \exists Q^{\prime} \cdot C[Q] \rightarrow Q^{\prime} \simeq_{\kappa} P^{\prime}, \text { and } \\
& C[Q] \rightarrow Q^{\prime} \Rightarrow \exists P^{\prime} . C[P] \rightarrow P^{\prime} \simeq_{\kappa} Q^{\prime}
\end{aligned}
$$

- For limit $\lambda, P \simeq_{\lambda} Q$ if $\forall \kappa<\lambda . P \simeq_{\kappa} Q$ (i.e. $\left.\simeq_{\lambda}=\cap_{\kappa<\lambda} \simeq_{\kappa}\right)$.

Then we have: $\cap_{\kappa \in \text { Ord }} \simeq_{\kappa}=\left|\Im_{\nu}^{*}\right|$.

The proposition tells us that the asynchronous convergence, together with the reductionclosure property, fully characterises the canonical theory. Note that the theory thus characterised is obtained without presupposing any specific convergence notion, not to say " $\Downarrow "$ above. Apart from this, what we have obtained is the theory in the line of applicative bisimulation in [1], but closed under all possible branching of multi-step reduction, thus incorporating the statefulness.

\section{Reduction Theories and Bisimilarities}

4.1. $\Im_{\nu}^{*}$ and equators. In the following we try to explicate the equations by $\Im_{\nu}^{*}$ in its difference from $\Im_{\approx_{a}}$, a theory based on bisimilarity we introduced in Section 2. As we noted in $3.2, \Im_{\approx_{s}}$ and $\Im_{\approx_{a}}$ are both sound, therefore are subtheories of $\Im_{\nu}^{*}$. The fact gives us a tractable way of equating terms in $\Im_{\nu}^{*}$. Moreover $\Im_{\approx_{a}}$ is based on asynchronous observables, and includes $\Im_{\approx_{s}}$ in its equations (i.e. $\left|\Im_{\nu}^{*}\right| \supset \approx_{a} \approx_{s}$ ). So a natural question is whether there are any pairs of terms not equated by $\Im_{\approx_{a}}$ but equated by $\Im_{\nu}^{*}$. Actually such pairs exist, and, interestingly, the additional equations capture one of the essential aspects of name passing operation in the asynchronous regime. It is related with equators in 2.1: by a chain of equators, which continuously forward messages, we can abstract locations. 
To clarify the essential point, we construct a small $\nu$-theory using this special agent. Let us write $P \stackrel{v \cdot w}{\leftrightarrow} Q$ for $P\{v / w\} \equiv Q\{v / w\}$, i.e. $P$ and $Q$ only differ in their (free) occurrences of $v$ and $w$. We define:

$$
\Im_{\mathcal{E} Q} \stackrel{\text { def }}{=}\{(P, \mathcal{E Q}(v w))=(Q, \mathcal{E Q}(v w)) \mid v, w, P, Q \text { arbitrary as far as } P \stackrel{v \cdot w}{\leftrightarrow} Q\}+.
$$

We can show that $\Im_{\mathcal{E} Q}$ is reduction-closed and isolates a set $\{P \mid P \rightarrow \stackrel{\uparrow a}{\uparrow}\}$ for each $a$. This shows that $\Im_{\nu}^{*}+\Im_{\mathcal{E} Q}$ is a sound theory, thus we have:

\section{PROPOSITION $4.1 \Im_{\mathcal{E} Q}$ is a subtheory of $\Im_{\nu}^{*}$.}

By the proposition, we know $\Im_{\nu}^{*}$ allows all the equations derivable in $\Im_{\mathcal{E} Q}$. Specifically:

CoROLlaRY $4.2 \Im_{\nu}^{*} \vdash(\mathcal{E Q}(v w), \leftarrow u v)=(\mathcal{E} \mathcal{Q}(v w), \leftarrow u w)$.

This explains why we only had observables which are without values to carry in the observability theorem. Since $\Im_{\approx_{a}} \forall(\mathcal{E} \mathcal{Q}(v w), \leftarrow u v)=(\mathcal{E Q}(v w), \leftarrow u w)$, together with Proposition 3.7, we obtain:

$\underline{\text { PROPOSITION } 4.3} \Im_{\approx_{i}}$ is a proper subtheory of $\Im_{\nu}^{*}$.

As to the exact difference between $\Im_{\approx_{a}}$ and $\Im_{\nu}^{*}$, we believe the following holds.

CONJECTURE $4.4 \Im_{\approx_{a}}+\{((\mathcal{E Q}(v w), \leftarrow u v)=(\mathcal{E Q}(v w), \leftarrow u w))\}$ axiomatises $\Im_{\nu}^{*}$

The task of proving (or disproving) the statement is left as a future exercise.

4. 2. Operators and observability. In this subsection we investigate whether there is any systematic way of regaining $\approx_{a}$ and $\approx_{s}$ by reduction-based formulation. The method provides an interesting correspondence between the basic operators in a given calculus, on the one hand, and induced canonical equations, on the other hand. The basic idea is to increase the power of agents to discern difference in behaviour by introducing additional syntactic operators.

To obtain $\approx_{a}$, we use the match operator [13]. It has the form "[u=v]", used as " $[u=v] P$ ", with a structural rule:

$$
[u=u] P \equiv P
$$

Note that the operator directly compares two names, so that it might well annihilate the power of the equators. We call the system extended with the new syntactic construct and the structural rule, $\nu=$. The new set of terms is denoted by $\mathbf{T}_{\nu=}$, which is a superset of $\mathbf{T}_{\nu}$. We use the same definitions for reduction and transition, written again as $\rightarrow$ and $\stackrel{l}{\longrightarrow}$. The asynchronous weak bisimilarity in this setting is written $\approx_{a}^{=}$. For this relation we have: 


\section{PROPOSITION $4.5 \approx_{\bar{a}}^{\bar{*}}$ is a congruence relation ${ }^{7}$.}

For equational theories, " $P=Q \Rightarrow[u=v] P=[u=v] Q$ " is added, and, using the incompatible pairs similar to those in Lemma 3.8, we can prove common isolation sets for the sound theories. This guarantees the existence of the maximum element, which we shall call $\Im_{\nu=}^{*}$. Regarding this, an interesting observability result is known (see [9]).

Proposition 4.6 Suppose $\Im_{\nu=}^{*} \vdash P=Q$. Then, with $l$ relevant w.r.t. $P$ and $Q$, whenever $P \stackrel{l}{\longrightarrow} P^{\prime} \Rightarrow Q \stackrel{l}{\Longrightarrow} Q^{\prime} \quad$ for some $Q^{\prime}$ with $P^{\prime} \approx_{a}^{=} Q^{\prime}$.

The proposition tells us strict semantic difference the operator induces in the calculus, as already suggested by Boudol [4]. In our context, the operator provides the capability to discriminate two locations (or names), which results in inability to abstract locations. Now Proposition 4.6 tells us $\approx_{a}^{\bar{*}} \supset\left|\Im_{\nu=}^{*}\right|$. But $\approx_{a}^{\bar{*}} \subset\left|\Im_{\nu=}^{*}\right|$ is easy using Proposition 4.5, so that one gets $\approx_{a}^{=}=\left|\Im_{\nu=}^{*}\right|$. Then, together with $\approx_{a}^{=} \cap\left(\mathbf{T}_{\nu} \times \mathbf{T}_{\nu}\right)=\approx_{a}$, which is easy, we obtain the following.

$\underline{\text { PROPOSITION } 4.7}$ (restoration of $\approx_{a}$ ) Let $\Im_{\nu=\mid T_{\nu}} \stackrel{\text { def }}{=}\left\{P=Q \mid \Im_{\nu=}^{*} \vdash P=Q \wedge\right.$

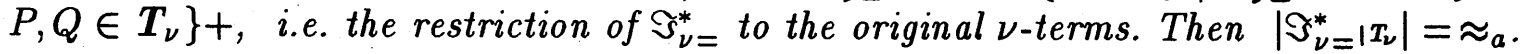

To obtain $\approx_{s}$, we first enrich the original $\nu$-calculus with capability of output synchronisation, which makes the system essentially the $\pi$-calculus in [14]. We add $\bar{u} v . P$ to the grammar, where the original $\leftarrow u v$ is embedded as $\bar{u} v . \Lambda$. We also replace (COM) in Definition 2.1 with $\tilde{c} \triangleright\left(\partial, \bar{u} v \cdot P, u x . Q, \partial^{\prime}\right) \rightarrow \tilde{c} \triangleright\left(\partial, P, Q\{v / x\}, \partial^{\prime}\right)$ and (REP) with $\tilde{c} \triangleright\left(\partial, \bar{u} v . P, ! u x . Q, \partial^{\prime}\right) \rightarrow \tilde{c} \triangleright\left(\partial, P, Q\{v / x\}, ! u x . Q, \partial^{\prime}\right)$. Then we easily obtain both input and output observables. However (and interestingly) we still cannot observe values in this framework, so that we again add the match operator, with which we finally restore $\approx_{s}$, by the same technical development as we have had for $\approx_{a}$. Thus the lattice of enrichment of operators results in the lattice of observables, hence of canonical equalities. The following picture depicts the situation. Below observables are listed on the left of the name of the system and the operators added to the base calculus are on the right ( " $\pi=$ " stands for the extension of the fragmentary $\pi$-calculus with match). A dotted line shows (upward) inclusion in canonical equations.

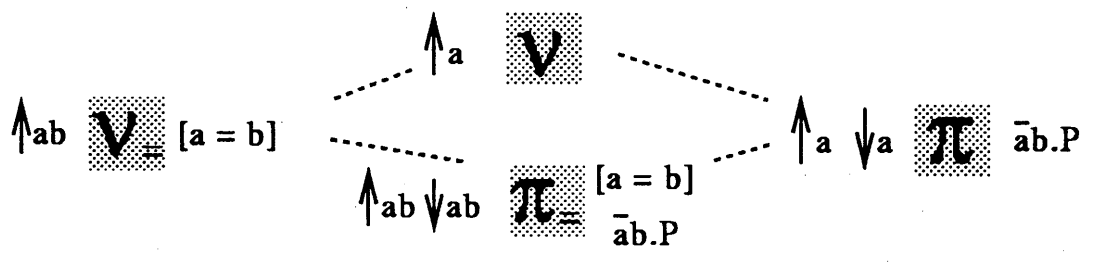

Note the picture arises uniformly from a single equational scheme: a deep study on operators and their semantic significance in process calculi is called for.

\footnotetext{
${ }^{7}$ This is mainly due to our proviso for bisimilarity over open terms in 2.2 , cf. [13].
} 
4. 3. Strong theories. Reduction theories are essentially incepted in the "weak" semantic framework, since they do not care the number of reduction steps, less the termination. Notably we have $\Omega=\Lambda$ in any sound theories. The following construction now tells us that we can easily "derive" strong theories out of them. In fact to any reduction theory a certain canonical strong theory corresponds to, and, as later to be seen, one such theory (derived from $\Im_{\nu}^{*}$ ) corresponds to a strong bisimilarity introduced in Section 2. The framework goes from the weak semantics to the strong semantics, which might be contrary to the usual practice: the idea here is to first try to capture the meaning of each term in the abstract setting, then refine the induced equations by considering exact steps a term need to reach possible semantic points. Cost is measured after sense is made, so to speak.

The basic definition of strong theories follows. Remember a theory is a subtheory of another theory if its equations are included in the latter's.

Definition 4.8 Given a reduction theory $\Im$, we say its subtheory $\Im^{\prime}$ is a strong reduction theory with a base $\Im$, if, whenever $\Im^{\prime} \vdash P=Q, P \rightarrow P^{\prime}$ implies, for some $Q^{\prime}$, $Q \rightarrow Q^{\prime}$ with $\Im^{\prime} \vdash P^{\prime}=Q^{\prime}$.

Put in other words, $\Im^{\prime}$ is a strong reduction theory if and only if, whenever $\Im^{\prime} \vdash P=Q$, $P \rightarrow^{n} P^{\prime}$ implies, for some $Q^{\prime}, Q \rightarrow^{n} Q^{\prime}$ with $\Im^{\prime} \vdash P^{\prime}=Q^{\prime}$, for all $n \in \omega$, where equations are only those allowed in its base. Thus a strong theory is derived from a base theory by "counting reduction steps". The following is important to get a (relatively) canonical strong theory from a base theory. The result is easy from Chain Lemma.

Proposition 4.9 Let $\Im_{i}$ be a strong theory whose base is $\Im$ for all $i \in I$. Then $\sum\left\{\Im_{i}\right\}_{i \in I}$ is also a strong theory with $\Im$ as its base.

Hence, given a reduction theory $\Im$, there is always the maximum strong theory with a base $\Im$. We also note that if the base is a strong theory, its maximum strong theory is the base itself. We call the maximum strong theory with the base $\Im_{\nu}^{*}, \Im_{\nu: s}^{*}$. For the theory, we have: if $\Im_{\nu: s}^{*} \vdash P=Q$ and $P \stackrel{l}{\longrightarrow} P^{\prime}$ with $l$ relevant w.r.t. $P$ and $Q$, then there exists $Q^{\prime}$ such that $Q \stackrel{l}{\longrightarrow}{ }_{a} Q^{\prime}$ and $P^{\prime} \sim_{a} Q^{\prime}$ (for establishment of the property, the reasoning on the number of reduction steps is crucial; see [9]). Thus $\left|\Im_{\nu: s}^{*}\right| \subset \sim_{a}$. But $\left|\Im_{\nu}^{*}\right| \supset \approx_{a} \supset \sim_{a}$, so that $\Im_{\sim_{a}}$ is a strong theory with a base $\Im_{\nu}^{*}$, i.e. $\left|\Im_{\nu: s}^{*}\right| \supset \sim_{a}$. Therefore:

$\underline{\text { PROPOSITION 4.10 }}\left|\Im_{\nu: s}^{*}\right|=\sim_{a}$.

It is easy to know that the strong theory corresponding to $\Im_{\approx_{a}}$ is again $\Im_{\sim_{a}}$. This is because some differences in weak theories depend on the use of additional reduction steps (e.g. equators): if these should be counted, the additional equations disappear. Hence restoration of values in messages without the match operator in $\Im_{\nu: s}^{*}$, leading to the coincidence of the "strong core" in two different weak theories. Finally we note that $\Im_{\sim_{s}}$ is easily obtained from $\Im_{\approx_{s}}$ : the reasoning parallels the case of $\Im_{\nu: s}^{*}$. 


\section{Discussion}

5. 1. Results on other calculi. CCS [12] is the process calculus based on dyadic synchronization as the basic communication primitive. The structural equivalence and reduction relation for the calculus naturally follow. One essential issue in constructing reduction theories for CCS is that the summation is problematic in weak congruent theories in general. However we restore weak bisimilarity $\approx$ in our purely equational setting by considering the maximum sound theory where congruence is understood in terms of reduction contexts (see 3.2 ). Then the maximum sound theory, denoted by $\Im_{c c s \mid+}^{*}$, coincides with $\approx$ via an observability theorem (cf. [16]). Finally the sound maximum strong theory whose base is $\Im_{c c s \mid+}^{*}$, coincides with the strong bisimilarity in [12].

Equational theories based on reduction are extensively studied in terms of $\lambda$-calculus [2]. Specifically $\beta$-equality is subsumed in our reduction-closure property as noted, not to say many of our formal constructions are inspired by $\lambda$-theories. A natural question is: can we get any (interesting) observables following our framework based on reduction-closure? It seems that soundness does not directly leads to observability, regarding strongly unsolvable terms $[17,1]$ as the insensitive terms. However if we also add identification of $\mathrm{PO}_{\infty}$ terms $[17,18]$, then we obtain the canonical theory after picking up head normal forms as generic observables, which coincides with $\mathcal{K}^{*}$, the theory of $D_{\infty}$. Note, with this canonical theory, we have restored $\beta$-equality, reinforcing our standpoint to regard $\beta$-equality as a special case of the reduction-closure property.

5. 2. Related work and open issues. There are two important precursors to our work. Boudol [4] studied (in)equation over essentially the same system as $\nu$-calculus based on Morris-like contextual precongruence. Milner and Sangiorgi [16] have shown that their construction called barbed congruence coincides with strong bisimilarity in the case of CCS, also referring to the results in the weak case. An apparent difference is that we do not positively stipulate a presupposed notion of convergence to derive a canonical equality, as opposed to two precursors. In this sense, it may be safe to say that the present construction depends on less subjective semantic commitment. Nevertheless we note that our approach, a posteriori, reveals the import of so-called convergence predicate in $[16,4]$, see e.g. Proposition 3.13 , by discovering such a notion without stipulating it beforehand. It is yet to see to what range of formal systems insensitivity-based approach is effective, and how such effectiveness is related to semantic aspects of given calculi. The result in 4.2 is suggestive in this aspect.

Another (and possibly more important) difference from other approaches lies in our crucial usage of reduction-closure. While [16] uses a closure property on reduction relation, the property is used rather as a "minimized bisimulation", together with the convergence notion, for a usual relation. Taking the union of all such relations, one takes the maximum congruence within it. In contrast, our formulation regards the closure property as the essential criteria for equality over stateful processes, just as $\beta$-equality over $\lambda$-terms. While to take the maximum congruence within non-congruent equivalence is already known in the case of observation congruence in CCS [12], we would like to note that the situation in [16] is quite different from [12]; the base relation, $\approx$, in [12] is behaviourally sound while this may not be the case in barbed bisimilarity in [16] (where $a .0$ and a.b.0 are equated), so that we cannot know, at least a priori, that taking the 
congruence within it is significant or not. More study is needed regarding the issue.

One remarkable point is the similarity in a way of deriving canonical congruences over processes between our construction and those found in $\lambda$-calculi $[2,1,18]$, as was already noted. What is common in these constructions is that the canonical equality is obtained as the maximum element of a sizable family of "meaningful" equalities. The method is not only mathematically elegant but also provides a tractable proof method (cf. development in 4:1). Mathematical canonicity may even suggest correspondence with important models of the concerned formalism; at least such are cases in strict and lazy $\lambda$-theories, though concrete results in the concurrency setting are still missing.

Finally, noting that reduction-closed equalities roughly correspond to bisimilarities, it is natural to ask what formulation would correspond to e.g. may and must equivalences [5]. We can use "saturated" insensitive terms i.e. the set $\left\{P \mid \Im_{\nu}^{*} \vdash P=\Lambda\right\}$, possibly combined with divergence notion. To find more direct ways of deriving such theories (i.e. without deriving $\Im_{\nu}^{*}$ first) remains as an important open question.

Acknowledgements. We thank Professors Cliff Jones and Robin Milner for fruitful discussions. We also thank anonymous referees and friends for providing valuable comments and pointing out errors. Our gratitude to Professor Mario Tokoro for his encouragement and support. The first author is partially supported by JSPS Fellowships for Japanese Junior Scientists.

\section{References}

1. Abramsky, S., The Lazy Lambda Calculus. ed. by D. Turner, Research topics in Functional Programming, Addison Wesley 1990.

2. Barendregt, H., The Lambda Calculus: Its Syntax and Semantics. North Holland, 1984.

3. Berry, G. and Boudol, G., The Chemical Abstract Machine. Proc. 17th. Annual Symposium on Principles of Programming Languages, 1990.

4. Boudol, G., Asynchrony and $\pi$-calculus, INRIA Report 1702, INRIA, Sophia Antipolis, 1991.

5. De Nicola, R. and Hennessy, M. Testing equivalences for processes, Theoretical Computer Science, 34(1), 1984.

6. Engberg, U. and Nielsen, M., A Calculus of Communicating Systems with Label Passing, Research Report DAIMI PB-208, University of Aarhus, 1986.

7. Honda, K., Two bisimilarities in $\nu$-calculus, submitted. Also as Keio CS report 92-002, 1992.

8. Honda, K. and Tokoro, M., An Object Calculus for Asynchronous Communication. Proc. ECOOP'91, LNCS 512, Springer-Verlag 1991.

9. Honda, K. and Yoshida, Y., Reduction-based Process Semantics, The full version as Keio CS technical report 94-002, February 1994. A preliminary version as Keio CS technical report 92-003, October 1992.

10. Honda, K. and Yoshida, Y., Combinatory Representation of Mobile Processes, Proc. 21th. Annual Symposium on Principles of Programming Languages, 1994.

11. Jones, C.B., A pi-calculus Semantics for an Object-Based Design Notation, Proc. CONCUR '93, LNCS 715, Springer Verlag, 1993.

12. Milner, R., Calculus of Communicating Systems. LNCS 92, Springer-Verlag, 1980.

13. Milner, R., Parrow, J.G. and Walker, D.J., A Calculus of Mobile Processes. Part I/II. ECS-LFCS-89-85/86, Edinburgh University, 1989. 
14. Milner, R., Functions as Processes. Proc. of ICA LP'90, LNCS 443, Springer-Verlag, 1990.

15. Milner, R., Polyadic $\pi$-Calculus: a tutorial. Logic and Algebra of Specification, SpringerVerlag, 1992.

16. Milner, R. and Sangiorgi, D., Barbed Bisimulation. Proc. of ICALP'92, LNCS 623, Springer-Verlag, 1992.

17. Longo, G., Set Theoretical Models of Lambda Calculus: Theory, Expansions and Isomorphisms, Annals of Pure and Applied Logic, 24:153-188, 1983.

18. Ong, C-H.L., The Lazy Lambda Calculus: An Investigation into the Foundation of Functional Programming, Phd thesis, Imperial College, 1988.

19. Park, D., Concurrency and Automata on Infinite Sequences. LNCS 104, Springer-Verlag, 1980.

20. Thomsen, B., A calculus of higher order communicating systems. Proc. 16th. Annual Symposium on Principles of Programming Languages, 1989. 